\title{
Bi-Objective Optimization for Vehicle Routing Problems with a Mixed Fleet of Conventional and Electric Vehicles and Soft Time Windows
}

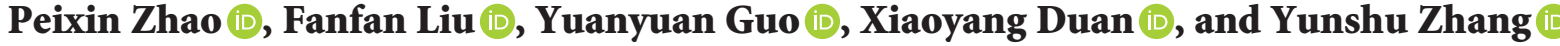 \\ School of Management, Shandong University, 27 Shanda Nanlu, Jinan 250100, Shandong Province, China \\ Correspondence should be addressed to Peixin Zhao; pxzhao@sdu.edu.cn
}

Received 6 June 2021; Accepted 14 August 2021; Published 6 September 2021

Academic Editor: Luca Pugi

Copyright @ 2021 Peixin Zhao et al. This is an open access article distributed under the Creative Commons Attribution License, which permits unrestricted use, distribution, and reproduction in any medium, provided the original work is properly cited.

\begin{abstract}
With the growing interest in environmental protection and congestion, electric vehicles are increasingly becoming the important transportation means. However, electric vehicles currently face several adoption barriers including high purchasing price and limited travelling range, so the fleets where electric vehicles and conventional vehicles coexist are closer to the current fleet management status. Considering the impact of charging facilities and carbon emission, this paper proposes a vehicle routing problem with a mixed fleet of conventional and electric vehicles and soft time windows. A bi-objective programming model is established to minimize total operational cost and time penalty cost. Finally, the nondominated sorting genetic algorithm II (NSGA-II) is employed to deal with this problem. Furthermore, single-objective optimization is carried out for the two objectives, respectively, and the linear weighting method is also used to solve the problem. Through the contrast of these results and the NSGA-II results, the effectiveness of the algorithm in this paper is further verified. The results indicate that two objectives are contradictory to some extent and decision-makers need a trade-off between two objectives.
\end{abstract}

\section{Introduction}

Energy and environment are hot spots of global concern. Climate and environment problems caused by the greenhouse effect emerge endlessly, which greatly threaten the people's health and daily life. Statistics from the International Energy Agency show that transportation is one of the main sources of carbon emissions, accounting for $24.64 \%$ of the total carbon emissions [1]. With the increasing social attention to environmental issues such as polluting emissions, noise, and congestion, more and more regulations are enacted to control carbon emissions from the transportation industry.

Electric vehicles (EVs), which have only a small amount of greenhouse gas emissions and very little noise, are considered to be a green alternative to conventional vehicles (CVs) in many occasions. Many cities have started electric bus or taxi pilot projects, such as Tokyo, New York, Montreal, and Shenzhen. Shenzhen, in particular, has become the first fully electrified city for franchised bus vehicles. In airport ground support service, Civil Aviation Administration of China is gradually carrying out "oil-toelectricity" activities for airport ground special vehicles. In the logistics field, some enterprises have already begun to employ EVs in their last-mile distribution business, e.g., in the field of small parcel shipping [2] and fresh products distribution [3].

Success in replacing existing CVs with EVs depends on several important factors. In addition to securing the sufficient financial investment, government support policies (e.g., regulations and subsidies), sufficient charging infrastructure, and advanced battery technology are all critical to improve market acceptance of EV fleets [4]. However, EVs have been facing formidable challenges for the limited power density of batteries, such as limited travelling range. In recent years, the wireless power transfer (WPT) is introduced as an advanced technology of addressing this problem with the hope of increasing the popularity of EVs [5]. 
Although the performance of this technology is remarkable in terms of flexibility, security, and esthetics, it has not been widely promoted and used in the field of EVs due to its high cost of infrastructure. Therefore, the attention of this paper is focused on wired charging EVs. Due to the high purchase cost and limited travelling range, it is still difficult for EVs to completely replace CVs [6]. Recognizing that there are obstacles to deploying large-size EV fleet, most enterprises start to gradually transition from just a few EVs. Hence, mixed fleets where EVs and CVs coexist are closer to the current fleet management status. The development of environmentally friendly and efficient transportation and distribution systems is an important challenge.

The mixed fleet vehicle routing problem (MFVRP) is a special case of the heterogeneous vehicle routing problem (HVRP). However, the electricity constraints and charging decisions of EVs make the MFVRP considerably more complex. Cirović et al. [7] investigated the green vehicle routing problem with a heterogeneous fleet composed of environmentally friendly and unfriendly vehicles, taking into account the fact that logistics operators have a limited number of environmentally friendly vehicles. However, when defining the route, friendly and unfriendly vehicles are considered separately, and the energy supplement of the vehicles is not considered. By using the bi-objective mixed integer programming models, Eskandarpour et al. [8] and Ren et al. [9], respectively, studied the MFVRP without considering the recharging of EVs, but Ren et al. [9] considered the time window of the customer. Using an improved multidirectional local search algorithm, the former aimed at minimizing distance cost and carbon emissions, while the latter minimized pollution emissions and delay time through an improved variable neighborhood search algorithm. Lu et al. [10] addressed the problem of scheduling a taxi fleet with CVs and EVs, designed an innovative multilayer taxi-flow time-space network, and proposed two simple but effective decomposition-based heuristics to efficiently solve the problem with practical sizes. In their paper, the recharging time of EVs was set to a fixed value.

Goeke and Schneider [11], Masmoudi et al. [12], and Li et al. [13] considered the recharge plans of EVs in the same way when studying the MFVRP, with the constraints that EVs must be fully charged at the charging station. Among them, Li et al. [13] conducted sensitivity analysis on the fixed cost, variable cost, and travelling range of EVs and indicated that high fixed costs and low variable costs make EVs need long-distance distribution, which is restricted by the limited travelling range. In addition, electric vehicle routing problem with time window and mixed fleet based on the fully charged policy was examined by Li et al. [14]. An integer programming model is presented and the branch and price algorithm is developed to obtain the optimal solution. Sassi et al. $[15,16]$ had a more complicated consideration on the recharge plans of EVs; that is, the EVs can be partially recharged at the charging station. Hiermann et al. [17] also made the same assumption for charging and considered the time windows of customers. They rationally planned the routing of the mixed fleet including conventional vehicles, plug-in hybrid vehicles, and electric vehicles. Macrina et al. $[18,19]$, Mouhrim et al. [20], and Yu et al. [21] also supposed that EVs can be partially charged and further studied the mixed fleet vehicle routing problem with time windows. Paper [18] explicitly considered the restrictions on the pollution emissions of CVs and minimized the cost under the pollution emissions restrictions, and paper [19] innovatively added the acceleration and deceleration process of the EV to the energy consumption model to make further improvements. Mouhrim et al. [20] assumed that the energy consumption is related to the vehicle load and travel distance, and established a multiobjective programming model. Yu et al. [21] used the comprehensive modal emission model (CMEM), established a mixed integer programming model with the objective of minimizing the vehicles travelling cost and emissions, and solved this problem through an adaptive large neighborhood search heuristic. The relevant literature on the mixed fleet vehicle routing problem is shown in Table 1.

The literature on the VRP with a fleet composed of both EVs and CVs is limited (see Table 1 for a summary). Most early research paid less attention to charging facilities, and then some researchers incorporated the charging decision of EVs into the consideration and made further improvements on the charging assumptions. In the majority of the aforementioned works, the authors mainly focus on the single-objective models and seldom consider the carbon emissions of EVs or soft time windows. Therefore, a Mixed Fleet Vehicle Routing Problem with Soft Time Windows (MFVRPSTW) considering carbon emissions of both EVs and CVs is proposed in this paper, and a bi-objective programming model is established to minimize the total operational cost and the time penalty cost. In contrast with previous studies, our work explicitly accounts for carbon emissions through taking it as a part of total cost. In order to solve the problem under investigation, we employ the nondominated sorting genetic algorithm II (NSGA-II), and numerical examples are given to verify the effectiveness of the algorithm.

This paper is organized as follows. In Section 2, we define the MFVRPSTW and its mathematical formulation. The NSGA-II is detailed in Section 3. The results of the computational experiments are provided in Section 4. Finally, Section 5 discusses the conclusions and directions for future research.

\section{Mathematical Formulation for MFVRPSTW}

2.1. Problem Statement and Assumptions. The enterprise dispatches vehicles from the distribution center to customers whose locations and time windows are known, and they return to the distribution center. The enterprise, which has the choice of EVs and CVs, requires each customer to be served and each vehicle to be dispatched only once. Due to the limited travelling range, the EVs can be recharged at any available charging stations. Referring to the EVs, CVs have a larger travelling range and fast energy replenishment speed and this process can be ignored. The relevant assumptions are as follows: 
TABLE 1: Summary of the literature on the mixed fleet vehicle routing problem.

\begin{tabular}{|c|c|c|c|c|c|c|c|c|}
\hline References & $\begin{array}{c}\text { Single- } \\
\text { objective }\end{array}$ & $\begin{array}{c}\mathrm{Bi}^{-} \\
\text {objective }\end{array}$ & $\begin{array}{l}\text { Hard time } \\
\text { window }\end{array}$ & $\begin{array}{l}\text { Soft time } \\
\text { window }\end{array}$ & $\begin{array}{c}\text { Carbon } \\
\text { emission }\end{array}$ & $\begin{array}{c}\text { Energy } \\
\text { consumption }\end{array}$ & Charging & Algorithm \\
\hline$[8]$ & & $\sqrt{ }$ & & & $\sqrt{ }$ & - & - & $\begin{array}{l}\text { Enhanced } \\
\text { MDLS }\end{array}$ \\
\hline [9] & & $\sqrt{ }$ & $\sqrt{ }$ & & $\sqrt{ }$ & - & - & Improved VNS \\
\hline$[10]$ & $\sqrt{ }$ & & $\sqrt{ }$ & & & Distance & $\begin{array}{l}\text { Fixed } \\
\text { time }\end{array}$ & Heuristic \\
\hline [11] & $\sqrt{ }$ & & $\sqrt{ }$ & & & CMEM & Fully & ALNS \\
\hline$[12]$ & $\sqrt{ }$ & & $\sqrt{ }$ & & & CMEM & Fully & HALNS \\
\hline$[13]$ & $\sqrt{ }$ & & & & & Distance & Fully & SS-MACO \\
\hline$[14]$ & $\sqrt{ }$ & & $\sqrt{ }$ & & & Distance & Fully & $\begin{array}{c}\text { Branch and } \\
\text { price }\end{array}$ \\
\hline$[15]$ & $\sqrt{ }$ & & & & & Distance & Partially & Heuristic \\
\hline$[16]$ & $\sqrt{ }$ & & & & & Distance & Partially & ITS-LNS \\
\hline$[17]$ & $\sqrt{ }$ & & $\sqrt{ }$ & & & Distance & Partially & Metaheuristic \\
\hline [18] & $\sqrt{ }$ & & $\sqrt{ }$ & & $\sqrt{ }$ & Distance & Partially & ILS \\
\hline [19] & $\sqrt{ }$ & & $\sqrt{ }$ & & & Improved CMEM & Partially & HLNS \\
\hline$[20]$ & & $\sqrt{ }$ & $\sqrt{ }$ & & $\sqrt{ }$ & Distance and load & Partially & - \\
\hline$[21]$ & $\sqrt{ }$ & & $\sqrt{ }$ & & $\sqrt{ }$ & CMEM & Partially & ALNS \\
\hline Our work & & $\sqrt{ }$ & & $\sqrt{ }$ & $\sqrt{ }$ & Distance and load & Fully & NSGA-II \\
\hline
\end{tabular}

(1) The numbers of EVs and CVs are sufficient, and all vehicles have the same capacity.

(2) The time window of each customer is determined and known. The time window is soft; that is, the vehicles that arrive in advance can provide services but have to pay the waiting cost, while late arrivals also have to pay the delay cost.

(3) The number and locations of charging stations are given. For simplicity, the capacity of each station is assumed sufficient to simultaneously charge multiple EVs.

(4) The batteries of the EVs are fully charged before leaving the distribution center. Each EV can be recharged at any level of remaining battery power during its journey, but it must be fully charged before it can leave the station.

(5) Regardless of whether it is an EV or a CV, its energy consumption is directly proportional to the travel distance and load.

2.2. Energy Consumption for the Vehicles. This paper supposes that the energy consumption of vehicles is related to travel distance and load. Xiao et al. [22] have concluded that the energy consumption rate can be considered as a load dependent function through the statistical analysis of relevant data. Without losing any generalization, dividing the vehicle's weight into two parts, the vehicle's no-load weight $Q_{0}$ and the carried load $X$, the energy consumption per unit distance $\rho$ can be formulated as a linear function dependent on load $X$ approximately as

$$
\rho(X)=a\left(Q_{0}+X\right)+b .
$$

Defining the capacity of the vehicle as $Q$, the energy consumption per unit distance at full load as $\rho^{*}$, and the energy consumption per unit distance at no load as $\rho_{0}$, it can be seen from formula (1) that $\rho_{0}=a Q_{0}+b, \rho^{*}=a\left(Q_{0}+\right.$ Q) $+b$, and

$$
a=\frac{\rho^{*}-\rho_{0}}{Q} \text {. }
$$

So $\rho(X)$ can be rewritten as

$$
\rho(X)=\rho_{0}+\frac{\rho^{*}-\rho_{0}}{Q} X .
$$

2.3. Mathematical Model. In this problem, let $C=\{1,2, \ldots$, $n\}$ denote the set of customers, and $V=V_{E} \cup V_{C}$ be the set of vehicles, where $V_{E}$ is the set of EVs and $V_{C}$ is the set of CVs. $F$ denotes the set of charging stations, and we also need copies of charging stations to account for multiple visits at the same station. Thus, let $F /$ be the set of all stations and their copies. For simplicity, let $N=C \cup F$. Vertices 0 and $n+1$ denote instances of the distribution center, and all routes start at 0 and end at $n+1$. Other sets are defined as follows: $N_{0}=N \cup\{0\}, N_{n+1}=N \cup\{n+1\}, N_{0, n+1}=N \cup\{0\}$ $\cup\{n+1\}, C_{0}=C \cup\{0\}, F_{0}^{\prime}=F \prime \cup\{0\}$. The parameters and variables used in the model are shown in Table 2.

Considering the charging constraints and soft time window constraints, the bi-objective mixed fleet vehicle routing optimization model is established as follows:

$$
\begin{aligned}
\min \mathbf{f}_{1}= & \sum_{k \in V} \sum_{j \in N} f^{k} x_{0 j}^{k}+\sum_{k \in V} \sum_{i \in N_{0}} \sum_{j \in N_{n+1}} c^{k} \rho_{k}\left(u_{j}^{k}\right) d_{i j} x_{i j}^{k} \\
& +c_{e} \sum_{k \in V_{E}} \sum_{i \in F_{1}} \sum_{j \in N_{n+1}}\left(Y-y_{i}^{k}\right) x_{i j}^{k}+c_{o} \sum_{k \in V} \sum_{i \in N_{0}} \\
& \sum_{j \in N_{n+1}} \varepsilon^{k} \rho_{k}\left(u_{j}^{k}\right) d_{i j} x_{i j}^{k},
\end{aligned}
$$

$\min \mathbf{f}_{2}=p_{e} \sum_{i \in C} \max \left(e_{i}-t_{i}, 0\right)+p_{l} \sum_{i \in C} \max \left(t_{i}-l_{i}, 0\right)$, 
TABle 2: Parameter and variable definitions of the model.

\begin{tabular}{lc}
\hline Notation & Description \\
\hline$f^{k}$ & Fixed cost of vehicle $k$ \\
$c^{k}$ & Unit energy cost of vehicle $k$ \\
$\varepsilon^{k}$ & $\mathrm{CO}_{2}$ emission coefficient of energy used by vehicle $k$ \\
$c_{e}$ & Charge cost per unit of electricity \\
$c_{o}$ & Carbon tax \\
$p_{e}$ & Waiting cost per unit time \\
$p_{l}$ & Delay cost per unit time \\
{$\left[e_{i}, l_{i}\right]$} & Time window at vertex $i, i \in C$ \\
$s_{i}$ & Service time at vertex $i, i \in C$ \\
$d_{i}$ & Demand of vertex $i, i \in C$ \\
$d_{i j}$ & Travel distance between vertices $i$ and $j, i, j \in N$ \\
$t_{i j}$ & Travel time between vertices $i$ and $j, i, j \in N$ \\
$Y$ & Battery capacity of EVs \\
$g$ & Recharging rate \\
$Q$ & Vehicle capacity \\
$\rho_{k}(x)$ & Energy consumption per unit distance when the load of vehicle $k$ is $x$ \\
$t_{i}$ & Decision variable specifying the time of arrival at vertex $i, i \in N$ \\
$u_{i}^{k}$ & Decision variable specifying the remaining load of vehicle $k$ when arriving at vertex $i, i \in N$ \\
$y_{i}^{k}$ & Decision variable specifying the remaining battery capacity of EV $k$ when arriving at vertex $i, i \in N$ \\
$x_{i j}^{k}$ & Bision variable specifying whether arc $(i, j)$ is travelled by vehicle $k, i, j \in N$
\end{tabular}

s.t. $\sum_{k \in V} \sum_{j \in N_{n+1}, i \neq j} x_{i j}^{k}=1, \quad \forall i \in C$,

$\sum_{j \in N} x_{0 j}^{k}=\sum_{j \in N} x_{j, n+1}^{k} \leq 1, \quad \forall k \in V$,

$\sum_{j \in N_{n+1}, i \neq j} x_{i j}^{k} \leq 1, \quad \forall i \in F \prime, \forall k \in V_{E}$,

$\sum_{i \in N_{0}, i \neq j} x_{i j}^{k}-\sum_{i \in N_{n+1}, i \neq j} x_{j i}^{k}=0, \quad \forall j \in N, \forall k \in V$,

$t_{i}+\left(t_{i j}+s_{i}\right) x_{i j}^{k}-M\left(1-x_{i j}^{k}\right) \leq t_{j}$,

$\forall k \in V, \forall i \in C_{0}, \forall j \in N_{n+1}, i \neq j$,

$$
\begin{aligned}
t_{i}+\left(\frac{\left(Y-y_{i}^{k}\right)}{g}+t_{i j}\right) & x_{i j}^{k}-M\left(1-x_{i j}^{k}\right) \leq t_{j}, \\
& \forall k \in V_{E}, \forall i \in F^{\prime}, \forall j \in N_{n+1}, i \neq j,
\end{aligned}
$$

$$
u_{j}^{k} \leq u_{i}^{k}-d_{i} x_{i j}^{k}+M\left(1-x_{i j}^{k}\right), \quad \forall k \in V, \forall i \in N_{0},
$$$$
\forall j \in N_{n+1}, i \neq j \text {, }
$$$$
0 \leq u_{j}^{k} \leq Q, \quad \forall k \in V, \forall j \in N_{0, n+1},
$$$$
y_{0}^{k}=Y, \quad \forall k \in V_{E} \text {, }
$$$$
0 \leq y_{j}^{k} \leq y_{i}^{k}-\rho_{k}\left(u_{j}^{k}\right) d_{i j} x_{i j}^{k}+M\left(1-x_{i j}^{k}\right) \text {, }
$$$$
\forall k \in V_{E}, \forall i \in C, \forall j \in N_{n+1}, i \neq j,
$$

$$
\begin{aligned}
0 \leq y_{j}^{k} \leq Y- & \rho_{k}\left(u_{j}^{k}\right) d_{i j} x_{i j}^{k}, \\
& \forall k \in V_{E}, \forall i \in F_{0}^{\prime}, \forall j \in N_{n+1}, i \neq j,
\end{aligned}
$$

$$
\begin{aligned}
& x_{i j}^{k} \in\{0,1\}, \quad \forall k \in V, \forall i, j \in N_{0, n+1}, i \neq j, u_{i}^{k}, y_{i}^{k}, t_{i} \geq 0, \\
& \forall k \in V, \forall i \in N_{0, n+1} .
\end{aligned}
$$

The objective function equation (4) includes four terms: the fixed cost of vehicles, the energy consumption cost of vehicles, the charging cost of EVs, and the cost of carbon emissions. Equation (5) represents the time penalty cost due to the waiting or delaying service of vehicles, which is used to measure the quality of service. Constraint (6) stipulates that each customer is visited exactly once, and constraint (7) guarantees that each vehicle is dispatched once at most and must return to the distribution center after completing its tasks. Constraint (8) ensures that each charging station can be visited multiple times by any EV, and constraint (9) is the flow conservation constraint. Constraints (10) and (11) restrict arrival time at successor vertices $j$ if the arc from $i$ to $j$ is traveled, and constraint (11) covers the case that the EVs visit the charging stations. Constraint (12) enforces the fulfillment of demand at customer vertices. Constraint (13) means that the remaining load of each vehicle does not exceed the capacity at any time. Constraints (14)-(16) are the energy constraints of EVs. Constraint (14) enforces that the EVs are fully charged when they depart from the distribution center. Constraints (15) and (16), respectively, indicate the remaining battery power constraints of successor vertices when the predecessor vertices are the customers and the charging stations. Finally, decision variables are defined in constraint (17).

\section{Algorithm for MFVRPSTW}

The traditional method of solving multiobjective model is to convert multiple objectives into a single objective. Like the linear weighting method, usually the decision-maker determines the weight coefficients of objectives in advance and integrates multiple optimization objectives into one objective. 
So the results obtained are subjective and cannot guarantee that multiple objectives are optimized at the same time. However, the method based on the Pareto optimal does not need to transform the objectives and can directly find the nondominated solution set, which is more widely used.

The nondominated sorting genetic algorithm (NSGA-II) proposed by Deb et al. [23] improved the selection process of individuals by employing the density value estimation strategy, fast nondominated sorting strategy, and elite strategy, which has been widely applied in various fields. This algorithm is one of the most popular multiobjective algorithms, which not only ensures the value of the optimal solution and obtains the Pareto front, but also reduces the complexity of the algorithm. Therefore, the performance of this algorithm is remarkable in terms of running speed and convergence, and we employ it to solve the problem under investigation.

\subsection{Algorithm Design}

3.1.1. Encoding. Each chromosome should carry information about the configuration of the fleet and the priority of the customer, so the chromosome is divided into two parts. The first part indicates the decision of fleet deployment, which is composed of $m$ genes ( $m$ is the number of vehicles). The value of the gene represents the type of vehicle, where 0 represents the EV and 1 represents the $\mathrm{CV}$. The second part is composed of $n$ genes ( $n$ is the number of customers), and the value of the gene indicates the customer number. For example, there are 3 vehicles in the distribution center to provide service for 5 customers, and the chromosome is shown in Figure 1.

3.1.2. Decoding. First assign the customer to the corresponding vehicle. Starting from the first gene of the second part of the chromosome, if the integer $a$ satisfies $\sum_{i=1}^{a} q_{i} \leq Q$ and $\sum_{i=1}^{a+1} q_{i}>Q, 0$ is inserted after the $a$-th gene, and then this process is repeated from the inserted 0 until the end of the chromosome. If the number of 0 inserted is less than $m-1$, we increase the number of 0 after the last 0 until its number is $m-1$, and finally, the head and the end of the second part will be inserted with a 0 , respectively. The genes between two 0 indicate the route sequence of a vehicle. Through the above process, an initial distribution plan is generated.

Secondly distinguish the type of vehicle and determine whether the EV needs to be charged. As shown in Figure 2, $R$ denotes the travelling range of the EV, and $S 1, S 2$, and $S 3$ are the distance between the customers $i$ and $j$, the distance between the customer $j$ and its nearest charging station, and the distance between the customer $i$ and its nearest charging station, respectively. If the remaining battery power cannot allow the EV to reach the nearest charging station after serving the next customer, the EV must be charged first to avoid stopping on the way when $R<S 1+S 2$. To ensure that the EV can reach the charging station, $R$ must be equal to or greater than the distance from the current customer to the nearest charging station. Therefore, when $S 3 \leq R<S 1+S 2$,

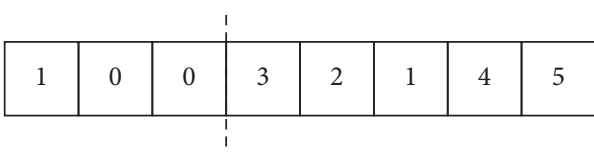

FIGURE 1: Representation of the chromosome.

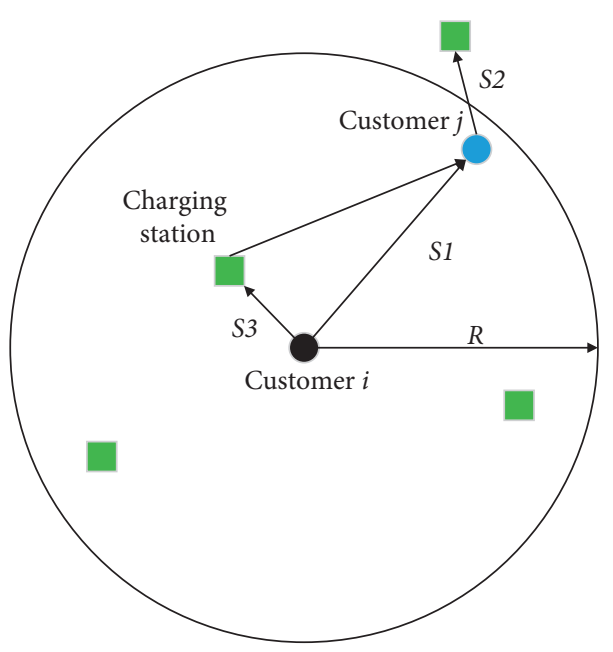

Figure 2: Schematic diagram of decoding.

the EV needs to recharge at the nearest charging station, and the charging station is inserted into its routing sequence.

Take the decoding result in Figure 3 as an example. The first vehicle is a CV with route $0-3-2-0$, which means that the $\mathrm{CV}$ departs from the distribution center, serves customers 3 and 2 , and finally returns to the distribution center. The second vehicle is an EV with route 0-1-4-6-5-0, which means after serving customers 1 and 4 the EV enters the charging station 6 for charging and then continues to serve customer 5. The third is an EV, but it is not used.

3.1.3. Population Initialization. The first part of the chromosome represents the type of vehicle, and 0 and 1 are randomly generated as the genes; the second part is realized by randomly arranging all customer numbers. Therefore, these two parts form an initial chromosome. A total of $2 \mathrm{~N}$ ( $N$ is the population size) individuals are generated by repeating the above process, and then the best $N$ individuals are selected as the initial population through nondominated sorting and crowding distance operators.

3.1.4. Selection, Crossover, and Mutation Operators. This paper employs the tournament selection method; that is, each time a certain number of individuals are drawn from the population, the best one is selected, and the operation is repeated until the given population size is reached.

The chromosome is designed to be divided into two parts in this paper, so crossover and mutation operations are performed on the two parts, respectively, and the crossover and mutation operators should be selected according to the different encoding methods. For crossover, the first part of the chromosome uses the single-point crossover, and the 


\begin{tabular}{|l|l|l|l|l|l|l|l|l|l|l|l|l|}
\hline 1 & 0 & 0 & 0 & 3 & 2 & 0 & 1 & 4 & 6 & 5 & 0 & 0 \\
\hline
\end{tabular}

FIGURE 3: Chromosome decoding result.

second part uses the partial-mapped crossover. As for mutation, we employ the two-point interchange mutation operator for both parts.

3.2. Steps of the Algorithm. The calculation procedure of this bi-objective optimization model is obtained based on the basic flow of NSGA-II in Figure 4:

(i) Step 1. Initialize the evolutional generation $n=0$, and generate an initial population $P_{n}$ with the number of individuals $N$.

(ii) Step 2. Generate the offspring population $Q_{n}$.

(iii) Step 3. Merge the parent and offspring populations; that is, $R_{n}=P_{n} \cup Q_{n}$.

(iv) Step 4. Calculate the nondominance rank and crowding distance of each individual in $R_{n}$ according to Steps 2.1 and 2.2, and select the best $N$ individuals to obtain the initial population of the next iteration $P_{n+1}$.

(v) Step 5. Determine whether the termination conditions for iteration are met. If so, stratify the population and output the individuals of the first layer; otherwise, go to Step 2.

\section{Numerical Examples}

4.1. Computational Results. In this section, the effectiveness of the algorithm is illustrated by three numerical instances. Referring to [11], this paper sets the number of charging stations as $\lfloor 0.1 \cdot|N|\rfloor$ ( $N$ is the number of customers). The instances used are generated based on the Solomon benchmark set, as shown in Table 3. Meanwhile, we assume that the number of vehicles is sufficient, so set the number of vehicles equal to the number of customers.

Since the final result will be affected by the location of the charging station, it is necessary to select the reasonable charging station locations by a location model.

$$
\begin{array}{ll}
\min & \sum_{i \in I} \sum_{j \in J} d_{i j} x_{i j}, \\
\text { s.t. } & \sum_{j \in J} x_{i j}=1, \quad \forall i \in I, \\
& x_{i j} \leq y_{j}, \quad \forall i \in I, j \in J, \\
& \sum_{j \in J} y_{j}=p, \\
& x_{i j}, y_{j} \in\{0,1\}, \quad \forall i \in I, j \in J,
\end{array}
$$

where $I$ is the customer set. Denote $J$ as the set of alternative charging stations. The decision variables are defined as follows:

$$
\begin{aligned}
& y_{j}= \begin{cases}1, & \text { if } j \text { is selected as the charging station, } \\
0, & \text { otherwise, }\end{cases} \\
& x_{i j}= \begin{cases}1, & \text { if customer } i \text { is served by charging station } j \\
0, & \text { otherwise. }\end{cases}
\end{aligned}
$$

In addition, the different vehicles have distinct energy consumption and carbon emissions. So papers $[6,24]$ are referenced to determine the relevant parameters of vehicle energy consumption. The $\mathrm{CO}_{2}$ emission factor $\varepsilon^{k}$ (according to the Intergovernmental Panel on Climate Change) is $0.785 \mathrm{~kg} / \mathrm{kWh}$ for EVs and $3.096 \mathrm{~kg} / \mathrm{L}$ for CVs. Other relevant parameters are shown in Table 4.

All experiments in this paper are based on MATLAB R2018b and implemented on a computer with a Core (TM) i5 processor $(1.6 \mathrm{GHz})$ and a memory of $16 \mathrm{~GB}$. The relevant parameters are set as follows: population size popsize $=100$, maximum number of iterations IterMax $=500$, crossover probability $p_{c}=0.9$, and mutation probability $p_{m}=0.1$.

The Pareto front obtained by 500 iterations of the algorithm for the three instances is shown in Figure 5. It is easy to know that the Pareto front slopes to the lower right; that is, the value of $f_{1}$ is getting smaller, while the value of $f_{2}$ is getting larger. All the solutions are not dominated by each other, which indicates that there is no positive correlation between the two objectives and they are contradictory to some extent.

4.2. Comparative Analysis and Discussion. In order to further verify the effectiveness of the NSGA-II, this paper firstly optimizes $f_{1}$ and $f_{2}$, respectively, by the classical genetic algorithm. The rationality of the Pareto solution set is further illustrated by comparing it with the optimal value of each objective.

Taking Instance I as an example, the optimization of $f_{1}$ is carried out and Figure 6(a) is obtained. The left picture shows that the optimal value changes with the number of iterations during the operation of the algorithm, which indicates that the algorithm is valid. The right picture is the routing of vehicles corresponding to the optimal solution (the triangles represent the distribution center, the dots denote the customers, and the squares are the symbol of the charging stations; the EVs are represented by dashed lines, while the solid lines represent the CVs). Figure 6(b) can be 


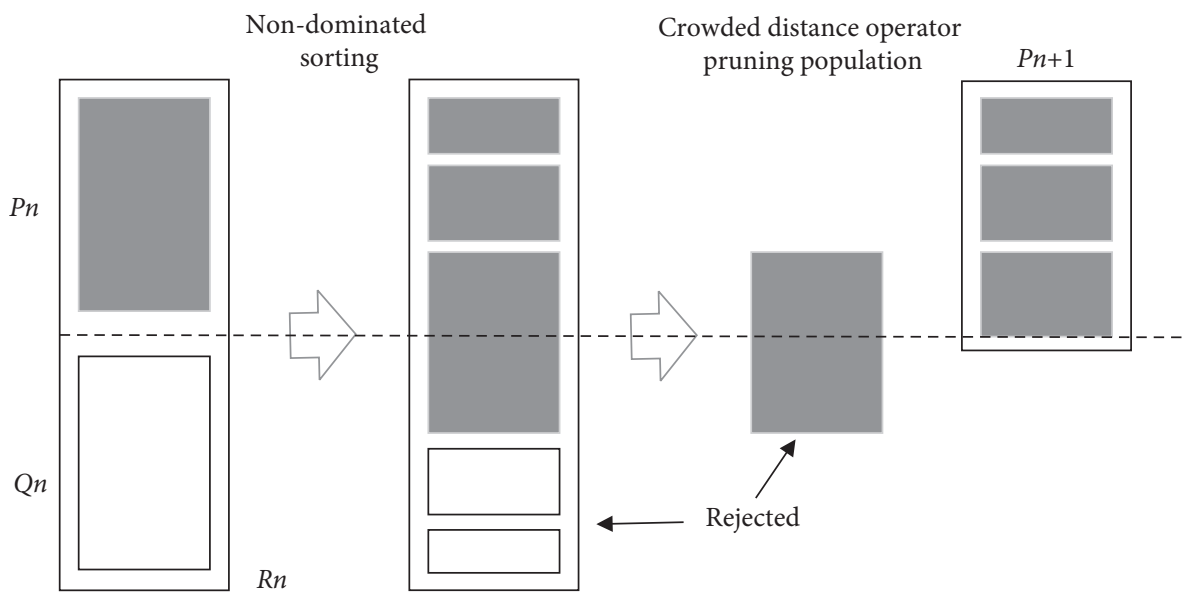

FIgURE 4: Basic flow of the NSGA-II algorithm.

TABLE 3: Number of customers and charging stations.

\begin{tabular}{lccc}
\hline & Instance I & Instance II & Instance III \\
\hline Number of customers & 25 & 50 & 91 \\
Number of charging stations & 2 & 5 & 9 \\
\hline
\end{tabular}

TABle 4: Values of parameters in the model.

\begin{tabular}{|c|c|c|}
\hline \multirow{2}{*}{ Notation } & \multicolumn{2}{|c|}{ Value } \\
\hline & EVs & CVs \\
\hline$f^{k}$ & 300 & 230 \\
\hline$c^{k}$ & 0.85 & 5.08 \\
\hline$\varepsilon^{k}$ & 0.785 & 3.096 \\
\hline$\rho_{k}(x)$ & $\rho_{k}(x)=0.5+(0.7 / 80) x$ & $\rho_{k}(x)=0.175+(0.225 / 80) x$ \\
\hline$c_{e}$ & & \\
\hline$c_{o}$ & & \\
\hline$p_{e}$ & & \\
\hline$p_{l}$ & & \\
\hline$Y$ & & \\
\hline$g$ & & \\
\hline Q & & \\
\hline
\end{tabular}

obtained by performing the same operation on $f_{2}$. The comparison of single-objective optimization and the Pareto solution set is shown in Table 5 .

As can be seen from Table 5 , when only optimizing $f_{1}$, the value of optimal solution is 2389.414 , which is better than all the solutions in Pareto solution set. However, the value of $f_{2}$ corresponding to this optimal solution is 1051.805 , which is worse than all the solutions in Pareto solution set. The same goes for the situation when $f_{2}$ is optimized. Therefore, if one objective is optimized separately, its optimal value is better than all the solutions of the Pareto solution set, while the other objective is worse than all the results of the Pareto solution set. This shows that the bi-objective optimization results obtained by the NSGA-II are reasonable.
On the basis of analysis about rationality, this paper compares with the linear weighting method to further analyze the validity of the results. Since $f_{1}$ and $f_{2}$ have the same dimension, they are given the same weight. $f_{1}+f_{2}$ can be regarded as one objective by adding directly, and its optimal solution can be obtained through genetic algorithm. The result is shown in Figure 6(c).

It can be concluded from the results in Table 5 that the value of the optimal solution obtained by the linear weighting method is 3030.94 (the corresponding values of $f_{1}$ and $f_{2}$ are 2483.145 and 547.795, respectively), and it is equal to the smallest value of $f_{1}+f_{2}$ in the Pareto solution set. Any solution in the Pareto solution set is nondominated by the optimal solution of the linear 


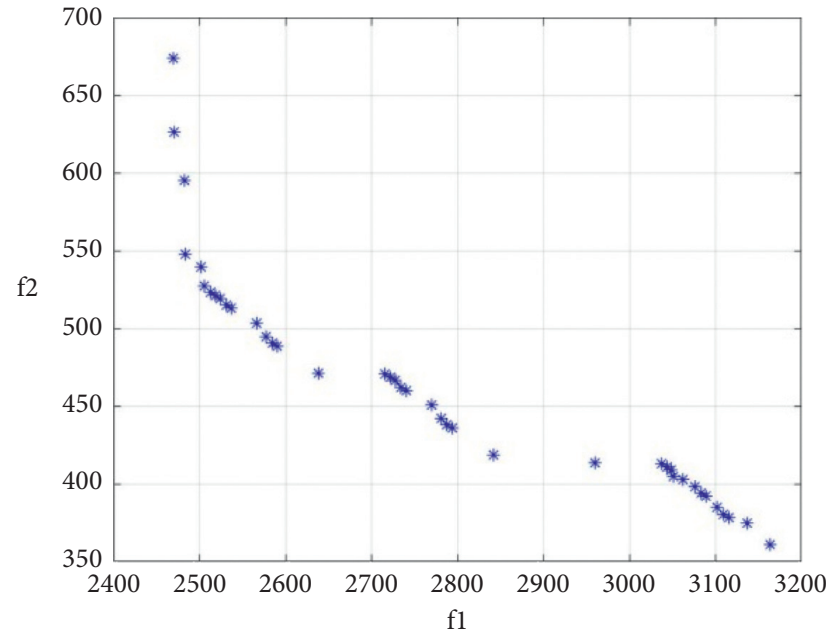

(a)

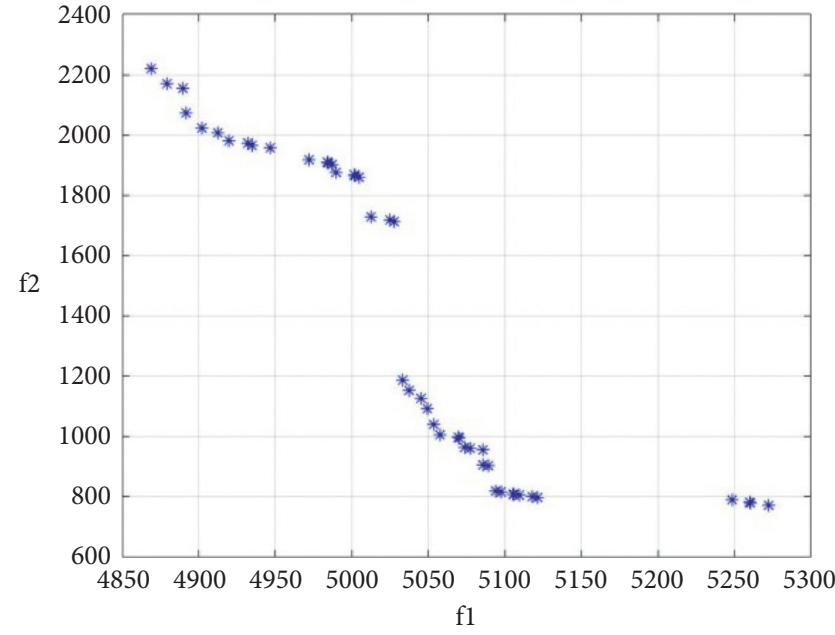

(b)

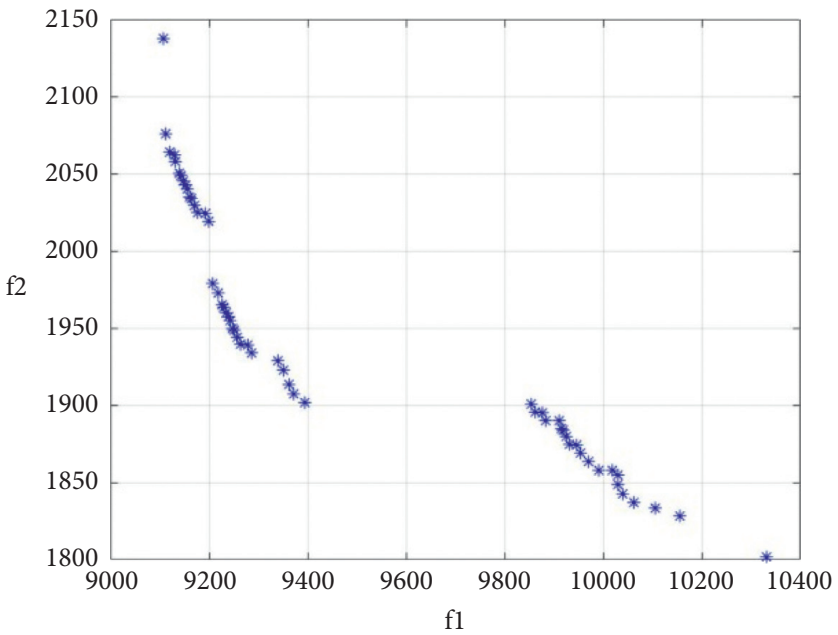

(c)

FIgURe 5: Pareto front. (a) Instance I. (b) Instance II. (c) Instance III.
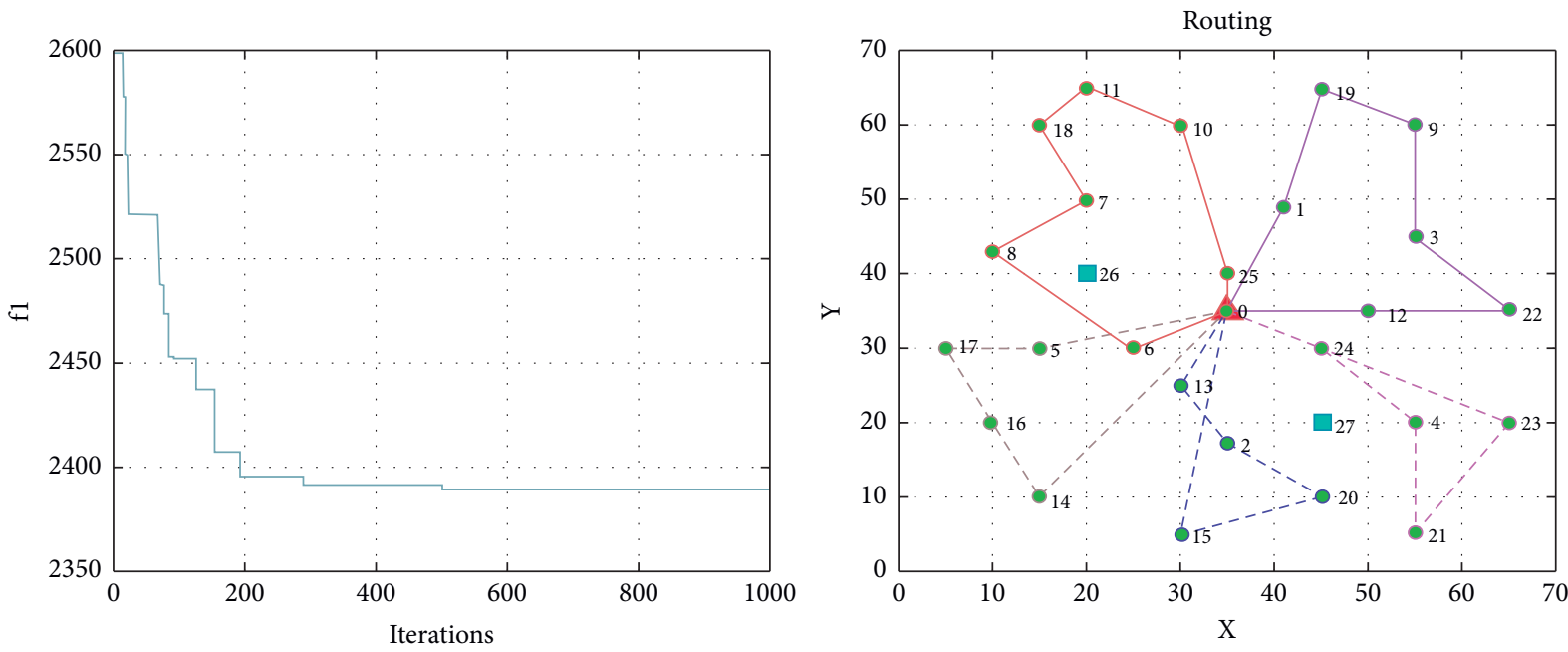

(a)

Figure 6: Continued. 

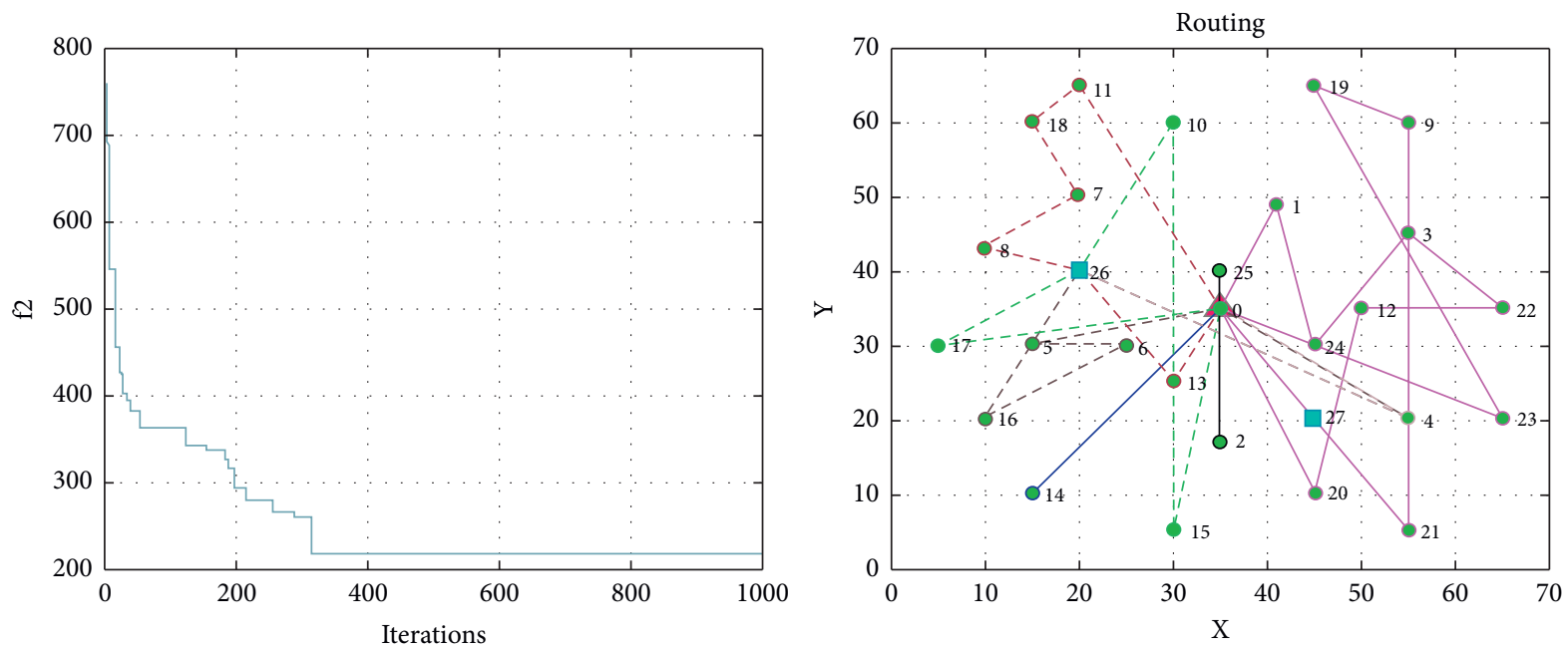

(b)
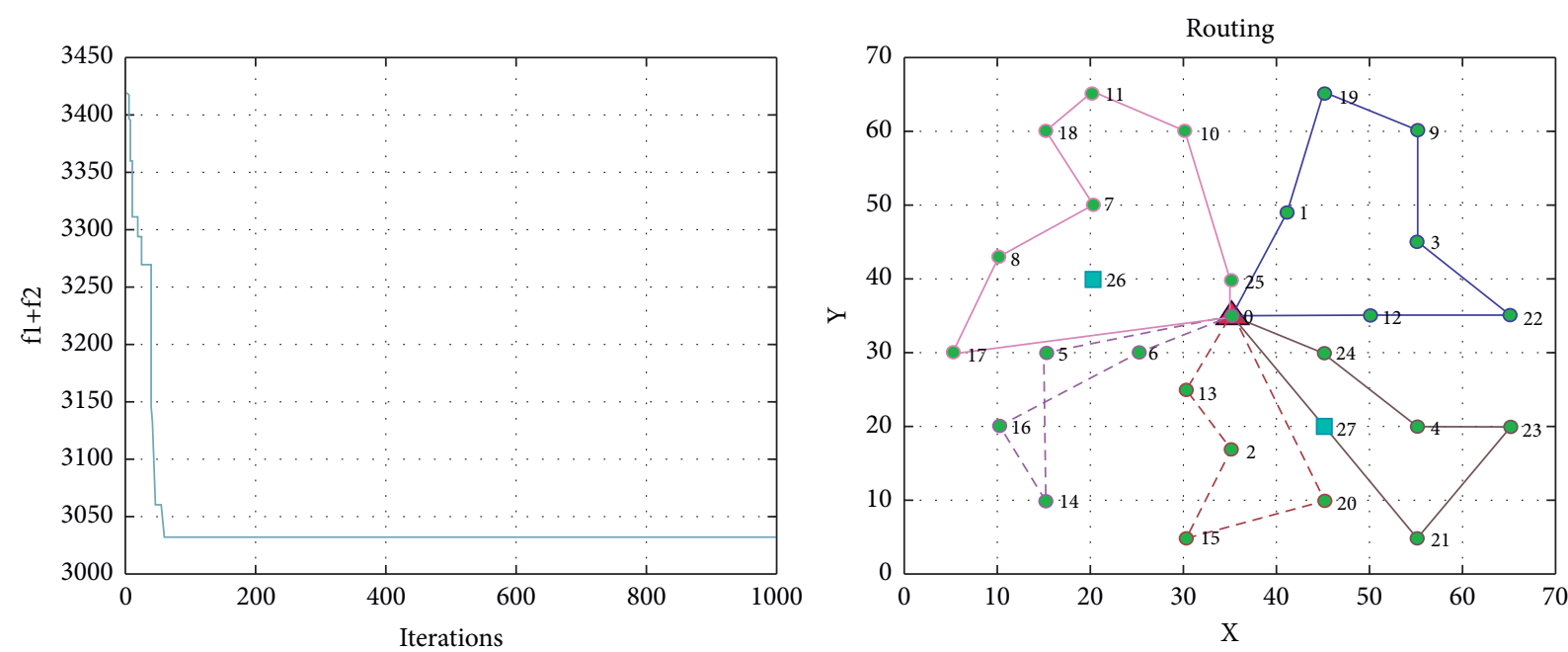

(c)

Figure 6: Single-objective optimization results of Instance I. (a) The results when only optimizing $f_{1}$. (b) The results when only optimizing $f_{2}$. (c) The results when optimizing $f_{1}+f_{2}$.

TABLE 5: Comparison of single-objective optimization and Pareto solution set for Instance I.

\begin{tabular}{|c|c|c|c|}
\hline & Solution & $f_{1}$ & $f_{2}$ \\
\hline \multirow{2}{*}{1} & The solution with the smallest $f_{1}$ in the Pareto solution set & 2469.860 & 674.201 \\
\hline & The optimal solution obtained when only optimizing $f_{1}$ & 2389.414 & 1051.805 \\
\hline \multirow{2}{*}{2} & The solution with the smallest $f_{2}$ in the Pareto solution set & 3163.406 & 360.986 \\
\hline & The optimal solution obtained when only optimizing $f_{2}$ & 3853.712 & 217.999 \\
\hline \multirow[b]{2}{*}{3} & The solution with the smallest $f_{1}+f_{2}$ in the Pareto solution set & 2483.145 & 547.795 \\
\hline & The optimal solution obtained when optimizing $f_{1}+f_{2}$ & 2483.145 & 547.795 \\
\hline
\end{tabular}

TABle 6: Comparison of single-objective optimization and Pareto solution set for Instance II and Instance III.

\begin{tabular}{|c|c|c|c|c|c|}
\hline \multirow{2}{*}{\multicolumn{2}{|c|}{$<!$ C Cnt:6> Solution }} & \multicolumn{2}{|c|}{ Instance II } & \multicolumn{2}{|c|}{ Instance III } \\
\hline & & $f_{1}$ & $f_{2}$ & $f_{1}$ & $f_{2}$ \\
\hline \multirow{2}{*}{1} & The solution with the smallest $f_{1}$ in the Pareto solution set & 4869.104 & 2219.836 & 9107.542 & 2137.740 \\
\hline & The optimal solution obtained when only optimizing $f_{1}$ & 4619.759 & 2508.618 & 8538.144 & 4890.566 \\
\hline \multirow{2}{*}{2} & The solution with the smallest $f_{2}$ in the Pareto solution set & 5272.350 & 771.920 & 10331.627 & 1801.689 \\
\hline & The optimal solution obtained when only optimizing $f_{2}$ & 7989.809 & 539.332 & 14335.238 & 1100.925 \\
\hline \multirow{2}{*}{3} & The solution with the smallest $f_{1}+f_{2}$ in the Pareto solution set & 5093.728 & 818.227 & 9120.650 & 2064.374 \\
\hline & The optimal solution obtained when optimizing $f_{1}+f_{2}$ & 5093.728 & 818.227 & 9120.650 & 2064.374 \\
\hline
\end{tabular}


weighting method, which indicates the effectiveness of NSGA-II.

The same operation is performed for Instances II and III and the results in Table 6 further verify the effectiveness of the NSGA-II.

\section{Conclusion}

Aiming at the coexistence of electric vehicles and conventional vehicles in the fleet, a new vehicle routing problem with mixed fleet and soft time window considering recharging is proposed, and a bi-objective mathematical programming model is established. Secondly, a novel coding and decoding method is designed according to the character of the problem, and the solution of the problem is obtained by NSGA-II. In order to illustrate the effectiveness of this algorithm, this paper further compares it with the singleobjective optimization and the linear weighting method. It shows that the results obtained by the NSGA-II are reasonable, and two objectives are contradictory to some extent. This paper can also provide management insights into the promotion of electric vehicles in other areas.

There are several interesting avenues for future research. For example, a more realistic modeling of the recharging process, the reduction of the battery capacity due to aging, and pickup and delivery simultaneously are all interesting topics.

\section{Data Availability}

The data used to support the findings of this study are available from the corresponding author upon request.

\section{Conflicts of Interest}

The authors declare that they have no conflicts of interest.

\section{Acknowledgments}

This work was supported by the National Natural Science Foundation of China (grant no. 72071122), Natural Science Foundation of Shandong Province (grant no. ZR2020MG002), and Social Science Planning Research Project of Shandong Province (grant no. 20CGLJ11).

\section{References}

[1] IEA, CO2 Emissions from Fuel Combustion, https://www.iea. org/subscribe-to-data-services/co2-emissions-statistics, 2020.

[2] P. R. Kleindorfer, A. Neboian, A. Roset, and S. Spinler, "Fleet renewal with electric vehicles at La poste," Interfaces, vol. 42, no. 5, pp. 465-477, 2012.

[3] J. Zhang, L. Shi, and Q. Wen, "Optimization of electric vehicle cold chain distribution route in charging mode of loading and unloading point," Manufacturing Automation, vol. 42, no. 7, pp. 92-96, 2020.

[4] J. Kim, S. Lee, and K. S. Kim, "A study on the activation plan of electric taxi in Seoul," Journal of Cleaner Production, vol. 146, pp. 83-93, 2017.
[5] F. Corti, A. Reatti, A. Nepote et al., "A secondary-side controlled electric vehicle wireless charger," Energies, vol. 13, no. 24, 2020.

[6] B. A. Davis and M. A. Figliozzi, "A methodology to evaluate the competitiveness of electric delivery trucks," Transportation Research Part E: Logistics and Transportation Review, vol. 49, no. 1, pp. 8-23, 2013.

[7] G. Ćirović, D. Pamučar, and D. Božanić, "Green logistic vehicle routing problem: routing light delivery vehicles in urban areas using a neuro-fuzzy model," Expert Systems with Applications, vol. 41, no. 9, pp. 4245-4258, 2014.

[8] M. Eskandarpour, D. Ouelhadj, S. Hatami, A. A. Juan, and B. Khosravi, "Enhanced multi-directional local search for the bi-objective heterogeneous vehicle routing problem with multiple driving ranges," European Journal of Operational Research, vol. 277, no. 2, pp. 479-491, 2019.

[9] X. Ren, H. Huang, S. Feng, and G. Liang, "An improved variable neighborhood search for bi-objective mixed-energy fleet vehicle routing problem," Journal of Cleaner Production, vol. 275, Article ID 124155, 2020.

[10] C.-C. Lu, S. Yan, and Y.-W. Huang, "Optimal scheduling of a taxi fleet with mixed electric and gasoline vehicles to service advance reservations," Transportation Research Part C: Emerging Technologies, vol. 93, pp. 479-500, 2018.

[11] D. Goeke and M. Schneider, "Routing a mixed fleet of electric and conventional vehicles," European Journal of Operational Research, vol. 245, no. 1, pp. 81-99, 2015.

[12] M. A. Masmoudi, M. Hosny, E. Demir, and E. Pesch, "Hybrid adaptive large neighborhood search algorithm for the mixed fleet heterogeneous dial-a-ride problem," Journal of Heuristics, vol. 26, no. 1, pp. 83-118, 2019.

[13] Y. Li, P. Zhang, and Y. Wu, "Vehicle routing problem with mixed fleet of conventional and electric vehicles," Journal of Systems and Management, vol. 29, no. 3, pp. 522-531, 2020.

[14] D. LI, Y. Chen, and Z. Zhang, "A branch-and price algorithm for electric vehicle routing problem with time windows and mixed fleet," Systems Engineering-Theory and Practice, vol. 41, no. 4, pp. 995-1009, 2021.

[15] O. Sassi, W. R. Cherif, and A. Oulamara, "Vehicle Routing Problem with Mixed fleet of conventional and heterogenous electric vehicles and time dependent charging costs," International Journal of Mathematical, Computational, Physical, Electrical and Computer Engineering, vol. 9, no. 3, pp. 171-181, 2015.

[16] O. Sassi, W. R. Cherif-Khettaf, and A. Oulamara, "Iterated tabu search for the mix fleet vehicle routing problem with heterogenous electric vehicles," in Modelling, Computation and Optimization in Information Systems and Management Sciences, pp. 57-68, 2015.

[17] G. Hiermann, R. F. Hartl, J. Puchinger, and T. Vidal, "Routing a mix of conventional, plug-in hybrid, and electric vehicles," European Journal of Operational Research, vol. 272, no. 1, pp. 235-248, 2019.

[18] G. Macrina, L. Di Puglia Pugliese, F. Guerriero, and G. Laporte, "The green mixed fleet vehicle routing problem with partial battery recharging and time windows," Computers \& Operations Research, vol. 101, pp. 183-199, 2019.

[19] G. Macrina, G. Laporte, F. Guerriero, and L. Di Puglia Pugliese, "An energy-efficient green-vehicle routing problem with mixed vehicle fleet, partial battery recharging and time windows," European Journal of Operational Research, vol. 276, no. 3, pp. 971-982, 2019. 
[20] N. Mouhrim, A. El Hilali Alaoui, and J. Boukachour, "Vehicle routing problem with mixed fleet of electric and conventional vehicles under emissions allowances," in Proceedings of the 4th IEEE International Conference on Logistics Operations Management (GOL), April 2018.

[21] V. F. Yu, P. Jodiawan, and A. Gunawan, "An Adaptive Large Neighborhood Search for the green mixed fleet vehicle routing problem with realistic energy consumption and partial recharges," Applied Soft Computing, vol. 105, Article ID 107251, 2021.

[22] Y. Xiao, Q. Zhao, I. Kaku, and Y. Xu, "Development of a fuel consumption optimization model for the capacitated vehicle routing problem," Computers \& Operations Research, vol. 39, no. 7, pp. 1419-1431, 2012.

[23] K. Deb, A. Pratap, S. Agarwal, and T. Meyarivan, "A fast and elitist multiobjective genetic algorithm: NSGA-II," IEEE Transactions on Evolutionary Computation, vol. 6, no. 2, pp. 182-197, 2002.

[24] W. Feng and M. A. Figliozzi, "Conventional vs electric commercial vehicle fleets: a case study of economic and acin the USA," Procedia - Social and Behavioral Sciences, vol. 39, pp. 702-711, 2012. 